\title{
The Routing Algorithm Based on Fuzzy Logic Applied to the Individual Physiological Monitoring Wearable Wireless Sensor Network
}

\author{
Jie Jiang, Yun Liu, Fuxing Song, Ronghao Du, and Mengsen Huang \\ Department of Electronic and Information Engineering, Beijing Jiaotong University, Beijing 100044, China \\ Correspondence should be addressed to Yun Liu; liuyun@bjtu.edu.cn
}

Received 19 June 2015; Revised 9 October 2015; Accepted 13 October 2015

Academic Editor: Chin-Feng Lai

Copyright (C) 2015 Jie Jiang et al. This is an open access article distributed under the Creative Commons Attribution License, which permits unrestricted use, distribution, and reproduction in any medium, provided the original work is properly cited.

\begin{abstract}
In recent years, the research of individual wearable physiological monitoring wireless sensor network is in the primary stage. The monitor of physiology and geographical position used in wearable wireless sensor network requires performances such as real time, reliability, and energy balance. According to these requirements, this paper introduces a design of individual wearable wireless sensor network monitoring system; what is more important, based on this background, this paper improves the classical Collection Tree Protocol and puts forward the improved routing protocol F-CTP based on the fuzzy logic routing algorithm. Simulation results illustrate that, with the F-CTP protocol, the sensor node can transmit data to the sink node in real time with higher reliability and the energy of the nodes consumes balance. The sensor node can make full use of network resources reasonably and prolong the network life.
\end{abstract}

\section{Introduction}

The wearable wireless sensor network [1] is applied to monitor the physiological information (heart rate, blood oxygen, breath, blood pressure, body temperature, and so on) and movement information (the speed, gait, trajectory, and the consumption of energy in the sport) of human body and the external environment (such as temperature, humidity, gas composition, and location) dynamically and continuously for a long time.

Focus on the wearable wireless sensor network among bodies, the wearable sensor node which is placed on the mobile human bodies constitutes the sensor network, the network topology of which changes fiercely. The wearable wireless sensor network which is applied in the individual physiological information monitoring is currently in the early research. And because the mobility of the human body is bigger and the topology changes dramatically, these require that sensor nodes transmit data in real time with high reliability and the energy of the nodes consumes balance which can prolong the network life. Therefore, new requirements for traditional wireless sensor network routing protocol are put forward.
The typical protocol of wireless sensor network mostly takes into account single performance. Many papers make improvement for these protocols: some take into account reducing the time delay performance and some take into account the energy utilization performance to make the network balance. All of them rarely involve the multiobjective optimization problem. Which are important for the application of the wireless sensor network in the wearable field.

The main contributions of this paper and the future outlook are summarized as follows.

We give a design of hardware system which is used for individual physiological monitoring. It uses wireless sensor network technology to sense the individual physiological information and integrates PC and PAD to achieve longdistance medical care purposes.

Based on the system we design, we put forward F-CTP protocol taking into account multiobjective optimization problem which is based on the fuzzy logic algorithm. It uses the distributed dynamic optimization to ensure that the sensor node can transmit data to the sink node in real time with higher reliability and the energy of the node consumes balance. The sensor node can make full use of network resources reasonably and prolong the network life. 
It makes the wireless sensor network better applied to wearable physiological monitoring area. The achievements in this paper will be the basis for development of wearable wireless sensor network applied in hospitals, the army, community-oriented and so on.

The rest of this paper is organized as follows. The related work is presented in Section 2. Section 3 gives the hardware system designed for individual wearable wireless sensor network. In Section 4, we briefly explain the CTP protocol; then, we illustrate the F-CTP mainly including three parts. First, we describe several definitions for fuzzy algorithm. Then, we give the membership function of input and output parameters and give the fuzzy rules. At last, we give the defuzzification method to rank the candidate neighbor node. In Section 5, the performance of F-CTP is evaluated. At last, we summarized this paper in Section 6.

\section{Related Work}

The wearable sensor network is a new discipline in recent years, there are a lot of researches about wearable sensor network development platform at home and abroad; however, there are less researches on the wearable sensor network communication protocols.

Firstly, some research results about the wearable sensing development platform are listed below. BSN node [2], which is developed by Imperial College London, is suitable for the field of medical care. The hardware system is composed of CC2420 and MSP430F14 of TI Company and the software is running on TinyOS. CodeBlue system [3] developed by Harvard University, which is used in an emergency situation to provide wireless medical monitoring. It uses wireless sensor network technology to sense the relevant physiological information and integrate PC, PAD, and other monitoring medical facilities to achieve long-distance medical care purposes. In paper [4], J. P. Carmo and his companions developed the smart electronic clothing, which can collect the physiological data of human body and transmit it to the remote terminal and then carry on further processing and analysis on the remote terminal. In paper [5], S. Choi and his companions developed the new wearable heart electrical signal monitoring equipment which can monitor body information during the sleep; the device uses the conduction tissue and PVDF, which is harmless to the human body for long time use. In paper [6], Otto et al. developed the product that not only can collect and save the human body's ECG signal but also can obtain the human body's motion state through the sensor. Therefore, it is a kind of system which can simultaneously monitor the ECG and motion state information. Po et al's [7] invention utilizes a smart sensor to detect physiological signals such as ECG and heart rate. And it can transmit these signals to the terminal through Bluetooth. These systems use a new type of sensor to obtain the physiological signals of the human body; some of the systems also have the function of wireless transmission. The current research is to study the communication of wearable wireless sensor networks from the aspects of sensor nodes, remote communication, sensors, and wearable embedding method, and most of the theoretical model of communication is still unpractical and cannot get a good promotion. This paper gives a design of hardware system used for individual physiological monitoring which is realized. The communication protocol of the system is based on wireless sensor network; on the other hand, it integrates positioning module using the Big Dipper on the basis of physiological monitoring such as ECG status and gesture information.

At present, the research on the communication of wearable wireless sensor networks is mainly carried out from the aspects of sensor nodes, remote communication, sensors, and wearable embedding method; the research on communication protocol of routing layer is rare. Basically all the wearable wireless sensor network communication protocols are based on wireless sensor network. There are specific needs for a wireless sensor network in the field of wearable wireless sensor which is introduced in Section 1. But the existing literature has not yet been targeted at multiobjective optimization about delay, energy balance, and delivery reliability for the wearable wireless sensor network. In the case of [8], a kind of CTP routing algorithm based on energy consumption for wireless sensor network is proposed, which takes into account the remaining energy of the next hop neighbor nodes in the data packet transmission process to achieve dynamic equilibrium network energy consumption. Paper [9] proposes a new clustering algorithm based on genetic algorithm for load balancing in wireless sensor networks. In the literature [10], Ronghui et al. proposed a kind of approximate algorithm based on the delay and the cost of two targets for routing. Paper [11] proposes a kind of multiobjective route based on ant colony algorithm, which integrates energy consumption, time delay, and robustness and transmission efficiency. In paper [12], the FLCHE clustering routing protocol based on fuzzy logic method is proposed, which considers the residual energy, energy consumption rate, and the distribution density of nodes. In paper [13], an energy-saving routing architecture with a uniform clustering algorithm is proposed in this paper to reduce the energy consumption in wireless body sensor networks. Paper [1] introduces that Pareto multiobjective optimization method and ant colony algorithm are used to improve the reliability and real time routing protocol. This paper proposed a multiobjective routing algorithms based on fuzzy logic algorithm, which integrates energy consumption, time delay, and reliability for wearable wireless network which makes the wireless sensor network better applied to wearable physiological monitoring area.

\section{Individual Wearable Wireless Sensor Network Monitoring System}

This section describes the design of an individual wearable wireless sensor network monitoring system [7]. The system is composed of three parts: extension, relay, and host which, respectively, are the soldier wearable device, relay terminal, and central data receiving control platform. For example, for a team composed of several members, the team leader equipped with relay terminal is regarded as the sink node 


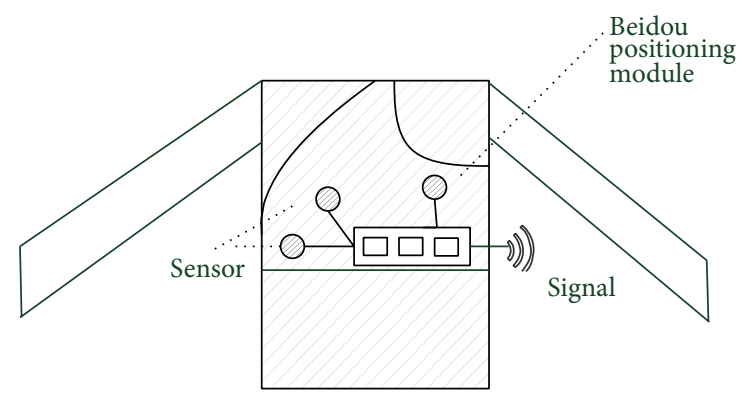

FIGURE 1: Appearance of the individual wearable device.

(Sink), which can display the monitoring data. Ordinary team members are equipped with wearable devices which are responsible for the collection of individual physiological information, attitude information (acceleration), and location information.

We build the wireless sensor network using Zigbee technology and use the CTP routing protocol (Collection Tree Protocol) to communicate between the sink node and sensor nodes. In order to guarantee the safety of communication, communication between the sensor nodes can be encrypted. The sensor node transmits its data or others nodes' data to the sink node by multihops transmission. The sink node collects all the data and then uploads them to a central data receiving control platform.

The individual wearable devices are placed on a wearable vest. The purpose is to monitor the individual position, gesture, and ECG status information in real time. The design principle is compact and easy to wear in the greatest extent to avoid the inconvenience of daily behavior. Appearance of the individual wearable device is shown in Figure 1.

The main module of individual wearable device includes WSN core module, BD positioning module, attitude sensor, physical sensor module, and battery module. The WSN core module consists of MSP430 microprocessor and CC2420 wireless transmission chip. The positioning part uses the BD2 positioning Module. The power management module includes lithium battery protection board, power indicator board, and boost regulator board to ensure the normal power supply. The structure diagram of the individual wearable device is shown in Figure 2.

\section{The Design of F-CTP}

4.1. The Classic CTP Routing Algorithm. CTP [14] is an aggregation protocol based on tree structure and sensor node delivery data to the sink node by anycast multihop. CTP uses the ETX value as the routing gradient, and the ETX value of sink node is 0 , the ETX of the other nodes is the ETX of its parent node plus the ETX of its parent link. The calculation of ETX is formulas (1) and (2). In formula (1), data_total represents the total packet delivered between two sensor nodes, and data_success represents the packet delivered successfully between two sensor nodes. In

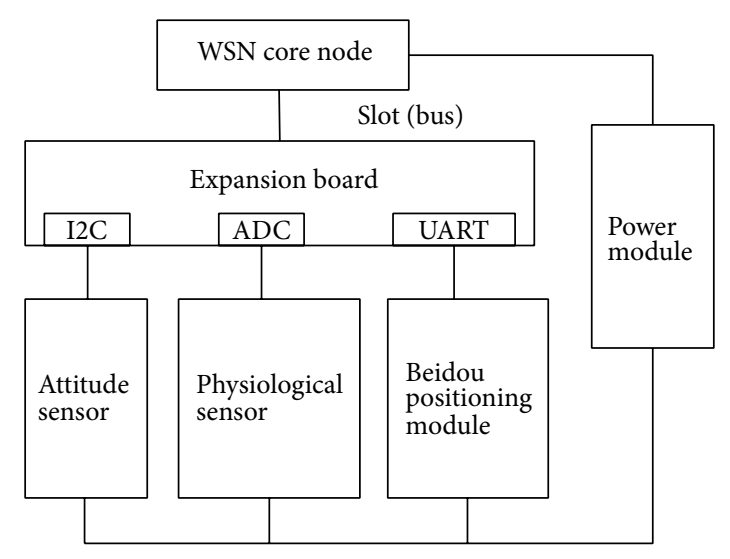

FIGURE 2: The structure diagram of the individual wearable device.

formula (2), ETX parent presents the ETX value of parent node, and ETX linkparent presents the ETX value of parent link:

$$
\begin{aligned}
\text { ETX } & =\left[\frac{\text { data_total }}{\text { data_success }}-1\right] \times 10, \\
\operatorname{ETX}(\text { node }) & =E T X_{\text {parent }}+E T X_{\text {linkparent }},
\end{aligned}
$$

where ETX(node) presents the ETX value of sensor node in wireless sensor network, when the nodes choose the path, they choose the path with the smallest ETX as the routing path. From formulas above, we can see that when the sensor node chooses the next hop the classic CTP only considers the packet transmission success rate, which is the reliability performance that we are concerned with.

4.2. A Routing Algorithm Based on Fuzzy Logic. For the requirements of the wearable wireless sensor network that is applied in the field of individual physiological monitoring, we propose a routing selection algorithm based on the fuzzy logic to improve the CTP protocol. The basic idea is calculating a reasonable value by fuzzy logic taking into account three parameters: reliability, time delay, and energy to replace the original ETX value. The routing algorithm can be divided into three phases. The first stage is defining the input and output parameters, respectively, and give the membership function which uses the language set to express the parameters. Then, fuse the language information using fuzzy rules and get the evaluation results of candidate parent node. At last, defuse the evaluation results by center of gravity method and choose the best path.

\subsubsection{The Description of the Input and Output Parameters.} The transmission model of the wireless networks is shown in Figure 3. We give some definitions as following.

Definition 1 (neighbor node set). The effective coverage range of the wireless signal from $A$ becomes its transmission region. The neighbors of $A$ are the nodes which fall into the transmission region of $A$. $B, C$, and $D$ are $A$ 's neighbors shown in Figure 3. 


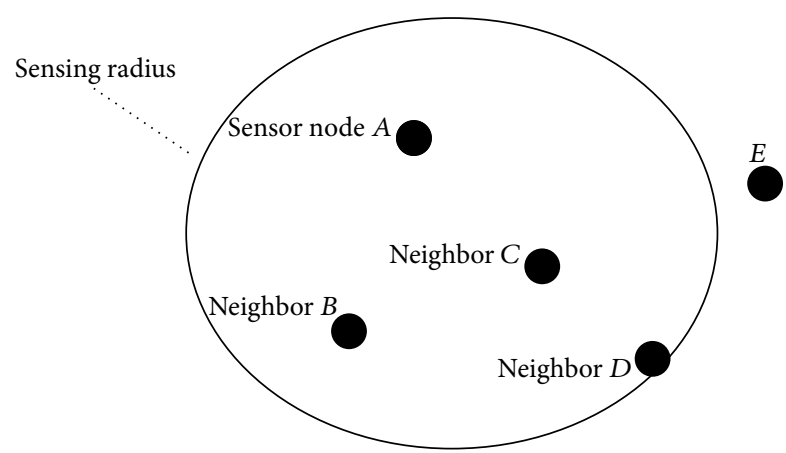

FIgURE 3: The transmission model for the sensor networks.

Definition 2 (the reliability parameter $R$ ). Calculate the probability of packets that are successfully transmitted between two sensor nodes. The formula is (2). Assume that $A$ sent $m$ packets to $B$ with a certain time, and the number of packets $B$ successfully received is $n$. Without considering the difference of two-way communication link, we assume that $R(A, B)=$ $R(B, A)$ :

$$
R(A, B)=\frac{n}{m}
$$

Definition 3 (time delay parameter $T$ ). Calculate the average time delay that $B$ successfully receives packets from $A$. The formula is (3). Assume that $A$ sent $n$ packets to $B$ continuously in a certain time; the number of the packet that $B$ successfully received is $n$. The send time series is $\left\{T_{1}, T_{2}, \ldots, T_{m-1}, T_{m}\right\}$ (the send time of $m$ packet) and the receiving time series is $\left\{T_{1}^{*}, T_{2}^{*}, \ldots, T_{m}^{*}\right\}$ (the receiving time of $n$ packet), ignoring the lost package:

$$
T(A, B)=\sum_{i \widehat{I n}}\left(T_{i}^{*}-T_{i}\right)
$$

Definition 4 (energy parameter $E$ ). Calculate the proportion of residual energy of one node accounted for the largest energy. The formula is (4). Assume that $E_{r}$ represents the residual energy of $B$ and $E_{\max }$ represents the largest energy among the neighbor nodes:

$$
E=\frac{E_{r}}{E_{\max }} .
$$

Definition 5 (one hop link quality table). A piece of entry in the routing table maintained by node $A$ is ( $A$, parent, $R(A, S)$, and $T(A, S))$. $A$ represents the current node, and parent represents a random candidate parent node among neighbor nodes. Parameter $E$ is not recorded in the routing entry, which is used for the calculation for routing selection.

4.2.2. Fuzzification. The membership functions and fuzzy rules are defined in Figure 4 and Table 1. The membership functions for the three input parameters $R, T$, and $E$ are defined according to our experimental data as (a), (b), and (c), based on output member function defined in (d).

Establish the appropriate fuzzy rules to fuse the three parameters and try to make the routing algorithm reach
TABLE 1: Fuzzy rules.

\begin{tabular}{lcccc}
\hline Rule & Reliability & Delay & Energy & Output \\
\hline 1 & High & Small & Enough & Perfect \\
2 & High & Small & General & Good \\
3 & High & Small & Few & Acceptable \\
4 & High & Medium & Enough & Good \\
5 & High & Medium & General & Acceptable \\
6 & High & Medium & Few & Unperfected \\
7 & High & Large & Enough & Acceptable \\
8 & High & Large & General & Unperfected \\
9 & High & Large & Few & Bad \\
\hline
\end{tabular}

the optimal evaluation. The fuzzy rules are composed of a series of fuzzy conditional statements in "IF-THEN" type, as in Table 1 . We use $3 * 3 * 3$ fuzzy rules. Part of the rules are shown in Table 1.

From the table, we can see the nodes with high reliability are more likely to be selected as the parent node. Nodes with lower delay also have a greater chance of being selected as the parent node. In addition, we also consider the energy index. The sensor node with more energy has more opportunities to be selected as the parent node. Comprehending three factors, we can find the F-CTP can select the optimal path.

4.2.3. Defuzzification. Center of gravity (COG) method is used to defuzzify the fuzzy result. Since the fuzzy logic can reconcile conflicting objectives, this step can provide a quick ranking of multiple candidates (neighbor nodes).

Each node maintains a routing table using the "IF-THEN" criterion. Each routing entry will output a representation with natural language, which is the fuzzy quantity. However, we really want to evaluate which routing entry is most perfect; we should change the fuzzy quantity as a clear number, which is called defuzzification. Here we select the center of gravity (COG) method for defuzzification, to get the accurate value of the probability of a candidate parent node becoming a parent node. So we can sort the probability of each routing entry; then, we can get the maximum value that is the optimal path.

\section{Performance Evaluation}

We assume there are only five nodes in the wireless sensor network and node 1 acts as a sink node. Besides, $(R, T)$ are parameters between each pair of nodes. The whole topology of the network is shown in Figure 5.

Parameters $(R, T)$ between nodes are recorded in routing table and the initial energy parameters are assumed to be the same. In addition, we further assume each node that sends and forwards a package consumes the same energy. After initial phase, each node maintains a routing table and the routing table of node 5 is shown in Table 2.

According to the classic CTP, we should choose entry 2 (red line) with highest reliability, which ignores the real time. With the routing algorithm based on fuzzy logic, we should choose entry 4 (blue line). We perform a further analysis 


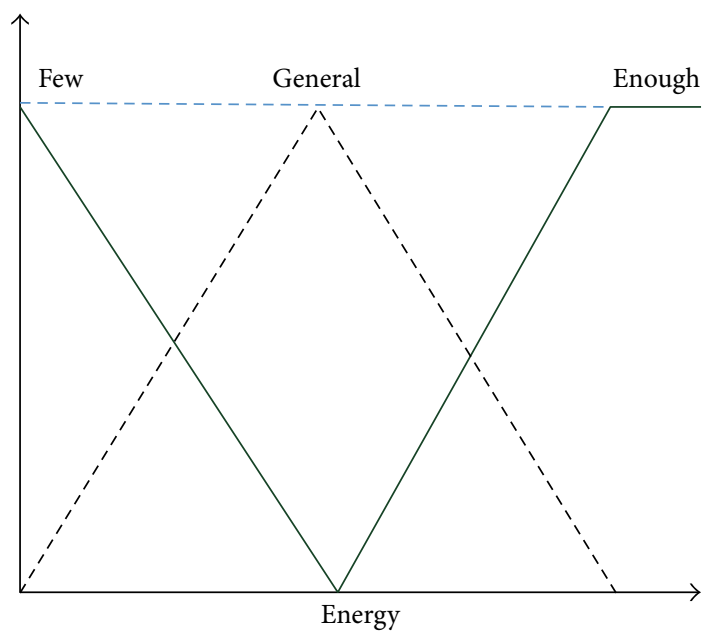

(a) The membership functions of $E$

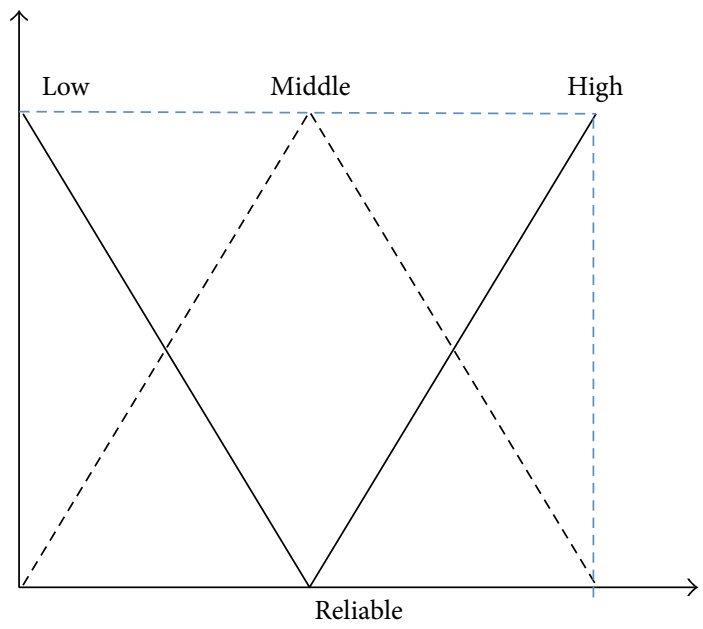

(c) The membership functions of $R$

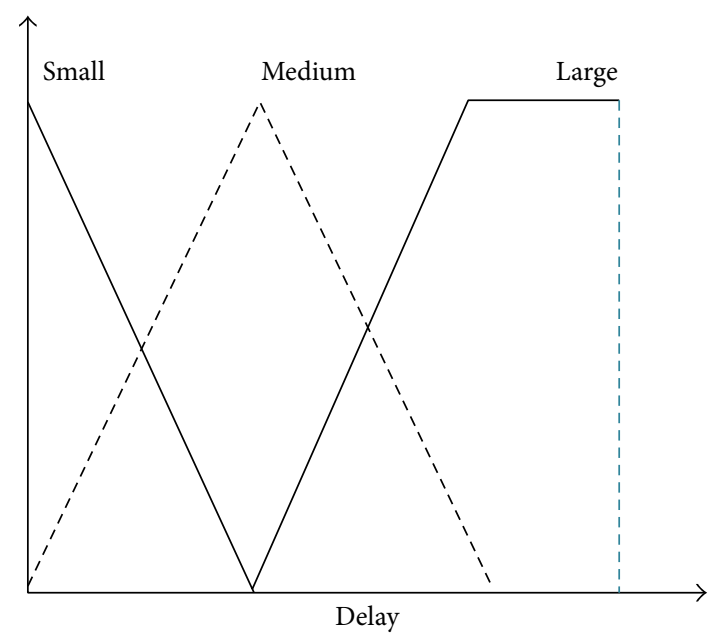

(b) The membership functions of $T$

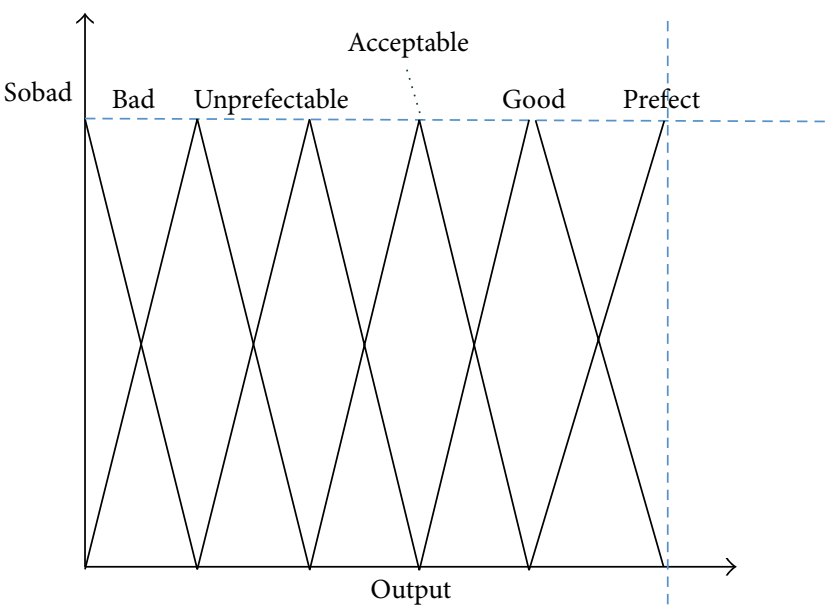

(d) The membership functions of output

FIGURE 4: The membership function of the input and output.

TABLE 2: Routing table of node 5.

\begin{tabular}{lcc}
\hline Current & Parent & $(R, T)$ \\
\hline 5 & 2 & $(0.36,50)$ \\
5 & 3 & $(0.48,90)$ \\
5 & 3 & $(0.288,100)$ \\
5 & 4 & $(0.432,20)$ \\
5 & 4 & $(0.2592,30)$ \\
\hline
\end{tabular}

of performance of the algorithm in case the network load increases. In the case, the number of data packets is 50 and 100 , we compare entries 2 and 4 of the performance of time delay and reliability.

With the increase of load, node consumes a certain amount of energy, under the condition of invariability of $R$ and $T$. When the 31st packet is transferred, because of the loss of node energy, the transmission path change to entry 1 . It proves that selection of transmission path will change with the increase of network load in the network environment, which reasonably uses the network resources, as is shown by the red line in the figure. The time delay and success ratio about classic CTP and F-CTP are shown in Figure 6 with the load of 50 and 100.

\section{Conclusion}

The fast growth of the biological physiological sensors, integrated circuit with low consumption, and the wireless communication makes the wearable wireless sensor network develop towards the individual physiological monitoring field. Routing Algorithm Research on wearable wireless network is the key to ensure the communication of nodes in the network. Therefore, the research of this paper will provide important evidence for the application of WSN in the individual physiological monitoring field.

This paper gives a design of hardware system used for individual physiological monitoring which integrates positioning module using the Big Dipper on the basis of physiological monitoring such as ECG status and gesture 


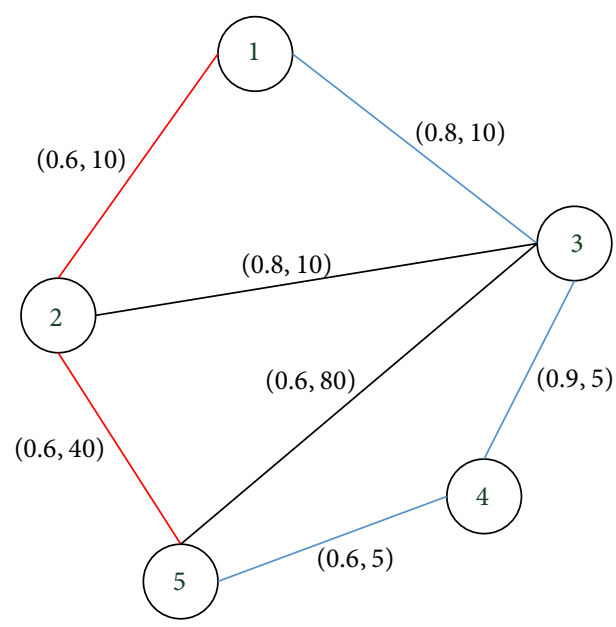

FIGURE 5: The topology of wireless sensor network for simulation.

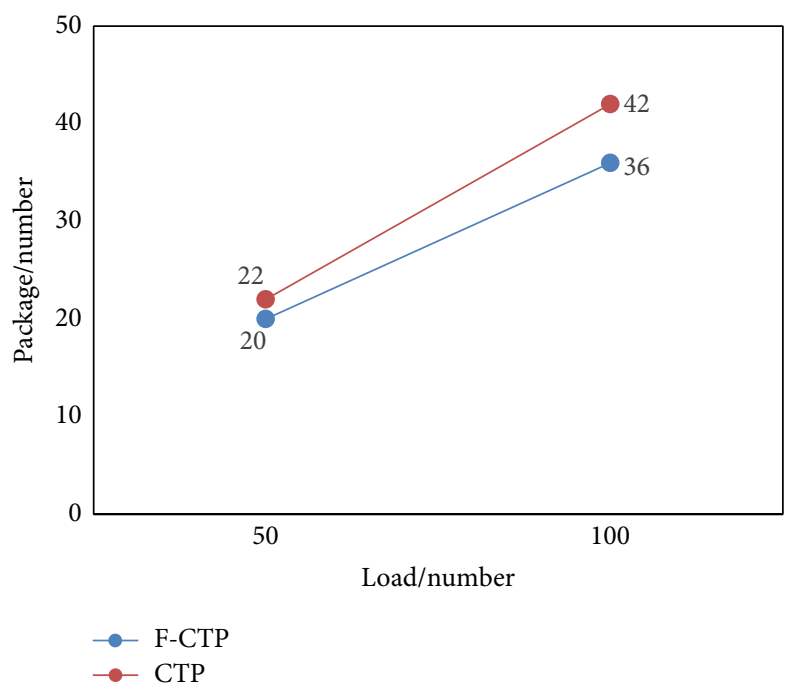

(a) Transmission quality comparison of different load cases

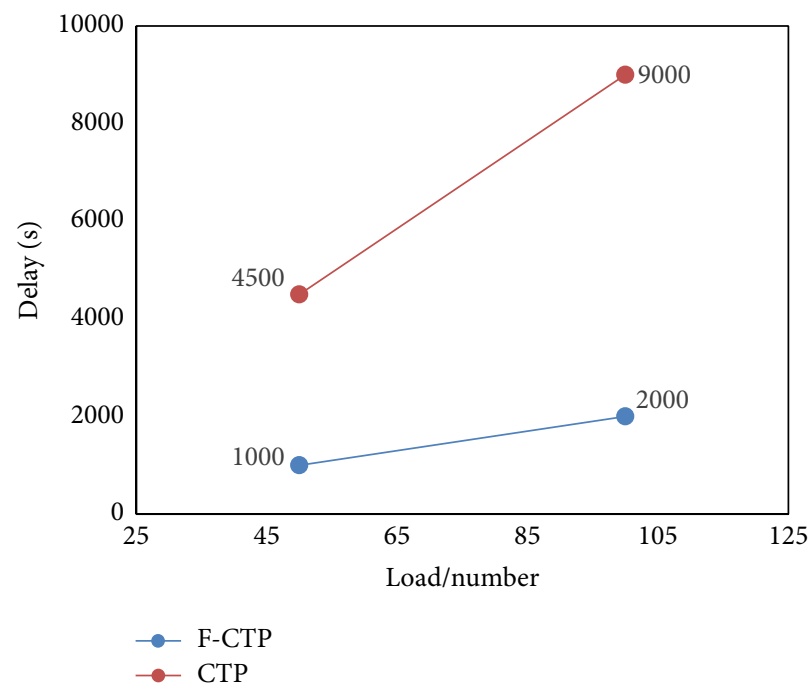

(b) Delay comparison of different load cases

FiguRE 6: The result of simulation. information. Multiobjective optimization of real time, reliability, and energy balance is one of the important research directions in the field of wearable wireless sensor. In this paper, we develop a routing algorithm using the fuzzy logic based on the CTP protocol, which is applied in the individual wearable wireless sensor network monitoring system. The improved F-CTP optimizes the selection of parent node, fully considering the time delay, packet success receiving rate, and residual energy, so that the whole network is more optimized and the life cycle of the whole network is effectively prolonged. But the problem is that our simulation is just a simple verification; the F-CTP put forward in this paper has not been applied in our hardware system and some membership functions used in fuzzy logic algorithms are also needed to be adjusted in more practice in the future research. Otherwise, a new heuristic algorithm can be given, which can search for a suitable routing path faster and better. We hope that wireless sensor network can be better applied to wearable physiological monitoring area.

\section{Conflict of Interests}

The authors declare that there is no conflict of interests regarding the publication of this paper.

\section{Acknowledgments}

This research is supported by Fundamental Research Funds for the Central Universities (2015YJS027) and National Natural Science Foundation under Grant 61371071.

\section{References}

[1] D. P. Quan, Wireless Sensor Network QoS Routing Studied for the Wearable Physiological Testing, Donghua University, 2012.

[2] B. P. L. Lo, S. Thiemjarus, R. King, and G.-Z. Yang, "Body sensor network-a wireless sensor platform for pervasive healthcare monitoring," in Proceedings of the 3rd International Conference on Pervasive Computing, Munich, Germany, 2005.

[3] M. Yu, S. J. Xiahou, and X. Y. Li, "A survey of studying on task scheduling mechanism for TinyOS," in Proceedings of the International Conference on Wireless Communications, Networking and Mobile Computing (WiCOM '08), pp. 1-4, IEEE, Dalian, China, October 2008.

[4] J. P. Carmo, P. M. Mendes, C. Couto, and J. H. Correia, " $2.4 \mathrm{GHz}$ wireless sensor network for smart electronic shirts," in Smart Sensors, Actuators, and MEMS II, vol. 5836 of Proceedings of SPIE, pp. 579-586, May 2005.

[5] S. Choi and Z. Jiang, "A novel wearable sensor device with conductive fabric and PVDF film for monitoring cardiorespiratory signals," Sensors and Actuators A: Physical, vol. 128, no. 2, pp. 317-326, 2006.

[6] C. Otto, A. Milenković, C. Sanders, and E. Jovanov, "System architecture of a wireless body area sensor network for ubiquitous health monitoring," Journal of Mobile Multimedia, vol. 1, no. 4, pp. 307-326, 2006.

[7] S. N. C. Po, G. Dagang, M. D. B. M. Hapipi et al., "Overview of MEMSWear II-incorporating MEMS technology into smart shirt for geriatric care," Journal of Physics: Conference Series, vol. 34, no. 1, pp. 1079-1085, 2006. 
[8] W. S. Zhu and W. Li, "The CTP algorithm based on energy awareness in wireless sensor network routing," Application Research of Computers, no. 11, 2014.

[9] P. Kuila, S. K. Gupta, and P. K. Jana, "A novel evolutionary approach for load balanced clustering problem for wireless sensor networks," Swarm \& Evolutionary Computation, vol. 12, pp. 48-56, 2013.

[10] H. Ronghui, K.-S. Lui, K.-C. Leung, and F. Baker, "An approximation algorithm for QoS routing with two additive constraints," in Proceedings of the IEEE International Conference on Network Protocols (ICNP '08), pp. 328-337, IEEE, Orlando, Fla, USA, October 2008.

[11] W. Wei and L. Zhi, "The multi-objective routing optimization of WSNs based on an improved ant colony algorithm," in Proceedings of the 6th International Conference on Wireless Communications, Networking \& Mobile Computing (WiCOM '10), pp. 1-4, IEEE, Chengdu, China, September 2010.

[12] G. D. Su and X. Wang, "The clustering routing protocol based on the fuzzy logical of wireless sensor network," Computer Application Research, no. 7, pp. 117-121, 2013.

[13] J.-Y. Chang and P.-H. Ju, "An energy-saving routing architecture with a uniform clustering algorithm for wireless body sensor networks," Future Generation Computer Systems, vol. 35, no. 3, pp. 128-140, 2014.

[14] The Collection Tree Protocol (CTP) [OL], 2010, http://www.tinyos.net/tinyos-2.1.0/doc/html/tep123.html. 

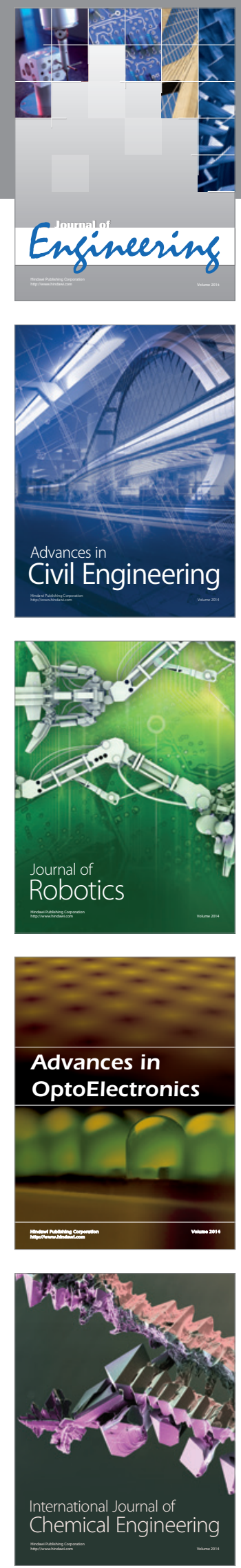

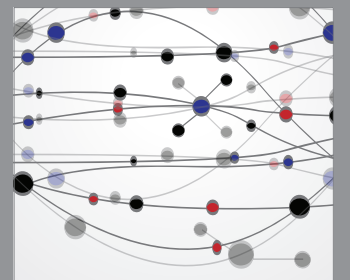

The Scientific World Journal
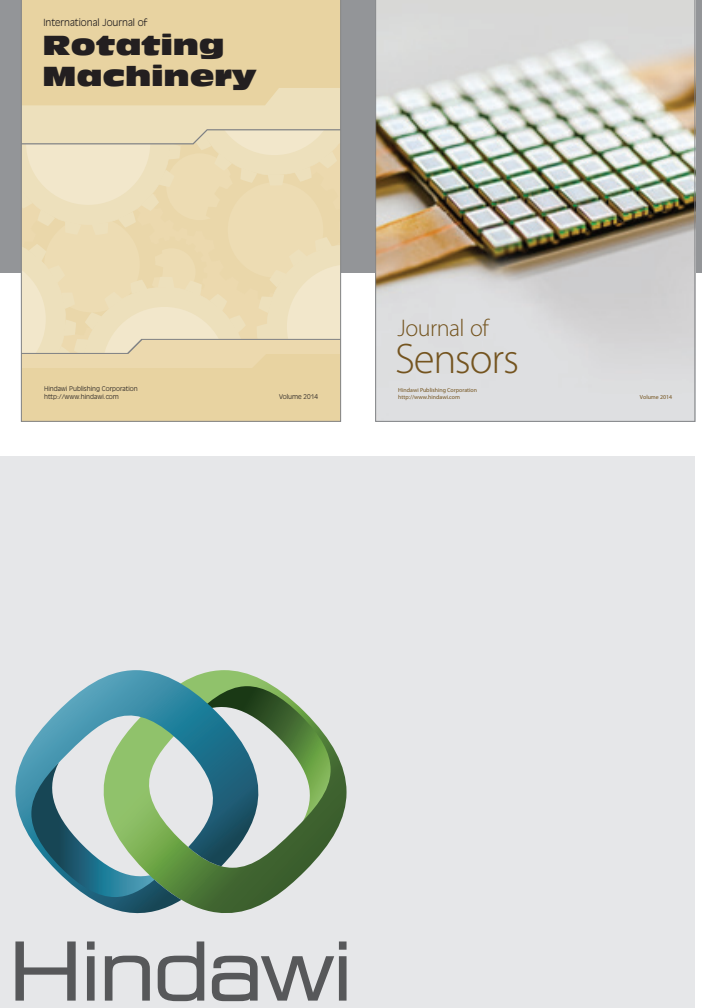

Submit your manuscripts at http://www.hindawi.com
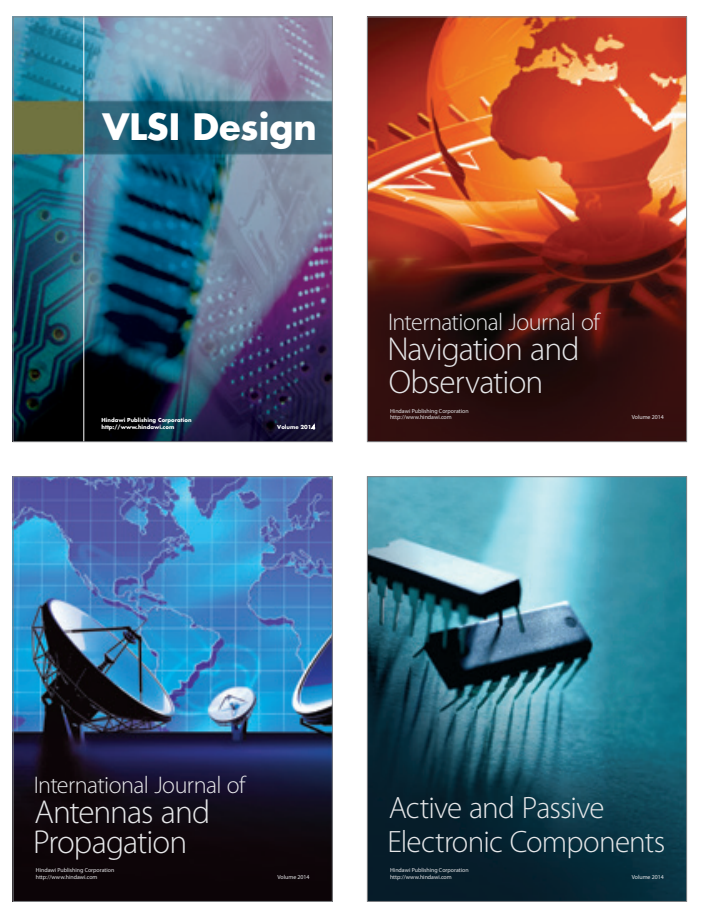
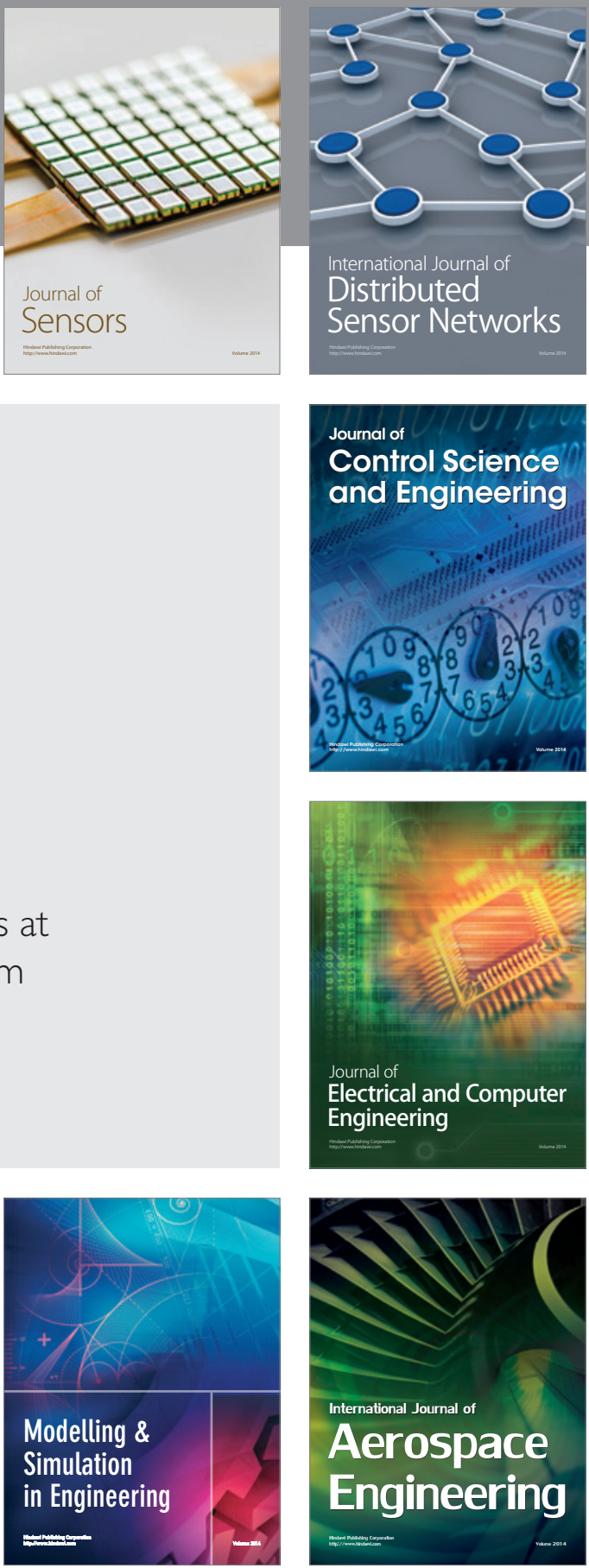

Journal of

Control Science

and Engineering
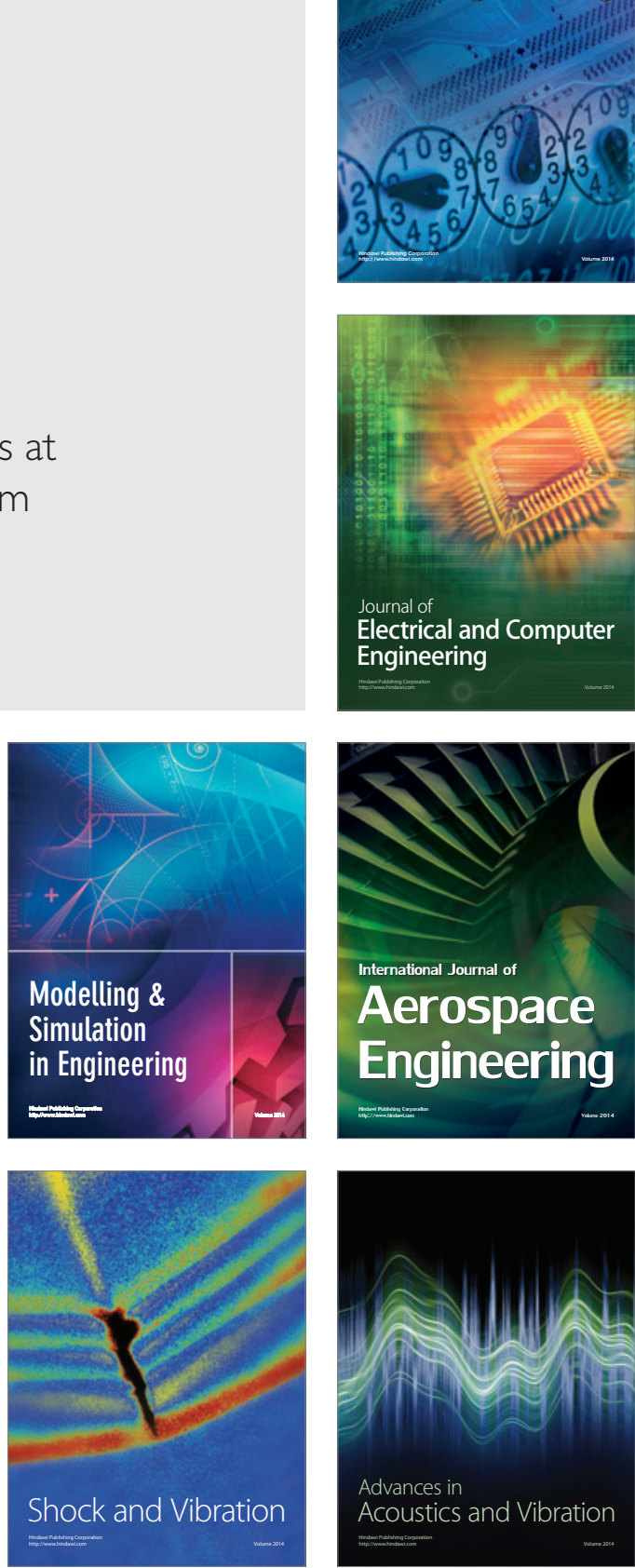